$\underline{\text { www.scielo.br/eq }}$

\title{
Estudo do comportamento eletroquímico do herbicida ametrina utilizando a técnica de voltametria de onda quadrada
}

\author{
M. F. Cabral ${ }^{1}$, D. de Souza', C. R. Alves ${ }^{1}$, S. A. S. Machado ${ }^{2}$ \\ ${ }^{1}$ Grupo de Biotecnologia de Alimentos e Ambiental - Centro de Ciências e Tecnologia - UECE - 60740-000 - Fortaleza - \\ CE-Brasil. \\ ${ }^{2}$ Grupo de Materiais Eletroquímicos e Métodos Eletroanalíticos - Instituto de Química de São Carlos - USP - 13560-960 - \\ São Carlos - SP - Brasil.
}

\begin{abstract}
Resumo: Foi realizado um estudo do comportamento eletroquímico do herbicida ametrina em meio ácido utilizando a técnica de voltametria de onda quadrada (SWV) combinada com eletrodo de gota suspensa de mercúrio (HMDE). O processo de redução da ametrina envolve a protonação prévia da molécula, seguido da transferência irreversível de dois elétrons com controle de velocidade dado pelo transporte de massa. A corrente de pico apresentou dependência linear com a concentração da espécie, $o$ que permitiu se calcular um limite de detecção de $0,1 \mathrm{mg} \mathrm{L}^{-1}(0,1 \mathrm{ppb})$ em amostras preparadas com água pura de laboratório, valor este que possibilita a aplicação desta metodologia eletroanalítica na análise de traços do herbicida em amostras ambientais.
\end{abstract}

Palavras-chave: ametrina; voltametria de onda quadrada; eletrodo de mercúrio.

\section{Introdução}

Apesar de toda a controvérsia sobre seus efeitos nocivos ao meio ambiente e, particularmente, ao homem, o uso de pesticidas tem aumentado intensamente nos últimos 50 anos, isto porque não existem muitas alternativas no controle das pragas ou das ervas daninhas que infestam as lavouras e que prejudicam a produção agrícola ou, até mesmo, no controle de vetores de doenças, como a doença de Chagas, a malária e a dengue. Desta forma, a produção mundial de pesticidas tem crescido continuamente, sendo a classe dos herbicidas, utilizados no combate a ervas daninhas, a que mais tem sido produzida.

Dentro desta classe, os herbicidas do grupo das triazinas é o maior, compreendendo cerca de $30 \%$ da produção mundial de herbicidas. Eles são utilizados no controle pré e pós-emergente de ervas daninhas nas mais variadas culturas, desde a década de 50. As s-triazinas normalmente possuem um anel heterocíclico de seis membros onde os átomos de carbono e nitrogênio são simetricamente localizados e os substituintes, localizados nas posições 2,4 e 6 , se constituem no diferencial entre as várias formulações disponíveis comercialmente [1].

A ametrina é uma s-triazina onde os substituintes são um grupo tiometil, um grupo etil e um grupo isopropil, formando a estrutura que leva o nome de N-etil-N-(1-metiletil)-6-(metiltio)-1,3,5 triazina 2,4-diamina. Na Figura 1 é apresentada a fórmula estrutural da ametrina. Ela é um herbicida sistêmico amplamente empregado em culturas de abacaxi, banana, café, cana-de-açúcar, citrus, milho, etc. Sendo razoavelmente estável, ela apresenta uma meia-vida de 20 a 100 dias, é muito móvel no ambiente e ainda altamente persistente em água e solo. Seus resíduos e metabólitos têm sido encontrados em águas subterrâneas mesmo depois de longos períodos após sua aplicação. A ametrina é muito tóxica e pode trazer sérios prejuízos à saúde e ao ambiente [2]. 


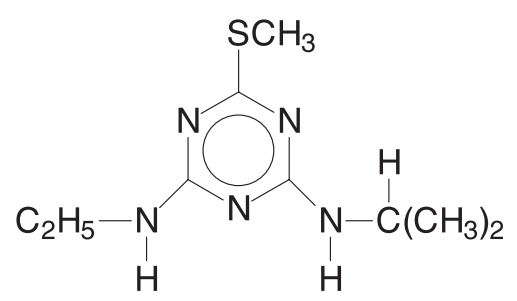

Figura 1. Fórmula estrutural da ametrina.

Assim como as demais s-triazinas, os métodos analíticos para a determinação da ametrina e seus produtos de degradação requerem a necessidade de técnicas de determinação de traços ou ultratraços ou ainda a análise de múltiplos analitos em matrizes complexas como amostras de águas e solos contaminados, onde a presença de outros compostos orgânicos exercem grande interferência. Desta maneira, o desenvolvimento de técnicas rápidas, baratas, sensíveis e que sofram a mínima interferência de outros compostos orgânicos, torna-se importante para a análise da ametrina e seus metabólitos, eventualmente presentes como contaminantes em água, solo e alimentos.

As técnicas eletroquímicas englobam todas estas características e além disto, a utilização de técnicas modernas de aplicação de pulsos de potencial possibilitam alcançar limites de detecção comparáveis àqueles obtidos nas técnicas cromatográficas, tradicionalmente aceitas como as técnicas padrão para estas análises. Além disto, as técnicas eletroquímicas tornam possível a aquisição de informações acerca do comportamento redox do sistema, tais como número de elétrons envolvidos na transferência eletrônica, tipo de transferência eletrônica, presença de adsorção de produtos e ou reagentes, e ainda informações sobre a cinética de transferência eletrônica e sobre o mecanismo da reação.

Desta maneira, a técnica eletroanalítica selecionada para o estudo do comportamento eletroquímico do herbicida ametrina foi a voltametria de onda quadrada (SWV - do inglês square wave voltammetry) uma técnica de pulso onde o pico de corrente resultante é proveniente da aplicação de pulsos de potenciais de altura $a$ (amplitude), que variam de acordo com um incremento de potencial, exemplificado como uma escada de potencial com largura $D E_{s}$ (incremento) e duração $2 t$ (período). As correntes elétricas são medidas de forma diferencial ao final dos pulsos e o sinal é dado como sendo a intensidade da corrente resultante. Por isto, o método apresenta excelente sensibilidade e baixa influência das correntes capacitivas [3].

As curvas de corrente-potencial apresentam perfil bem definido e são, geralmente, simétricas, porque as correntes são medidas somente no final de cada semiperíodo, e as variações na altura e na largura do pulso de potencial são sempre constantes, para um determinado intervalo de potenciais [4].

A utilização da SWV tem sido utilizada com sucesso para o estudo do comportamento eletroquímico e determinação analítica de pesticidas em diferentes amostras [5-8]. Assim, o objetivo deste trabalho foi estudar o comportamento eletroquímico do herbicida ametrina utilizando-se eletrodo de mercúrio e voltametria de onda quadrada e ainda se avaliar os limites de detecção em águas para futuras aplicações em amostras reais.

\section{Estado da Arte}

O primeiro trabalho utilizando técnicas eletroquímicas para determinação da ametrina foi realizado por Hance [9] em 1970. Utilizando a polarografia de corrente direta, ele observou que uma melhor resposta foi obtida em meio ácido, com um limite de detecção de $1,4 \times 10^{-6} \mathrm{~mol} \mathrm{~L}^{-1}(200 \mathrm{ppb})$.

Benadiková e colaboradores [10] utilizaram a voltametria de redissolução em conjunto com a voltametria de pulso diferencial para a determinação analítica da ametrina. Eles observaram que a corrente de pico aumentava linearmente com o tempo de acúmulo e com o potencial de acúmulo. Assim as condições otimizadas para a determinação da ametrina utilizando a redissolução foram: tampão $\mathrm{BR}$ pH 3,5; $\mathrm{t}_{\mathrm{acc}}=90 \mathrm{~s} ; \mathrm{E}_{\mathrm{acc}}=-01,02$ vs eletrodo saturado de calomelano. Nestas condições, o limite de detecção obtido foi de $7,89 \times 10^{-10} \mathrm{~mol} \mathrm{~L}^{-1}(0,179 \mathrm{ppb})$ para água pura e $1,00 \times 10^{-8} \mathrm{~mol} \mathrm{~L}^{-1}(3,0 \mathrm{ppb})$ para águas poluídas.

Ignjatovic e colaboradores [11] estudaram o comportamento polarográfico de algumas s- 
triazinas utilizando a polarografia de pulso diferencial. Para a ametrina eles observaram que em meio de tampão fosfato $\mathrm{pH}$ 3,0 ela apresenta um pico de redução em $-1,04 \mathrm{~V}$ vs SCE e uma relação linear entre a concentração de ametrina e a corrente de pico num intervalo de concentração de 0,005 a 5,0 mg L-1 (ppm). A aplicação desta metodologia a 20 amostras de águas com resíduos industriais mostrou limites de detecção que variaram de 0,09 a $345 \mathrm{mg} \mathrm{L}^{-1}$ (ppm), com a utilização da polarografia de pulso diferencial. Estes resultados foram comparados com os resultados obtidos por cromatografia gasosa com detector de ionização de chama que foram de 0,08 a $337 \mathrm{mg} \mathrm{L}^{-1}$ (ppm).

Este trabalho traz como inovação a utilização da SWV ao estudo eletroanalítico da ametrina. Esta metodologia possibilita uma varredura completa do potencial em curto intervalo de tempo, se comparado com as técnicas eletroanalíticas tradicionais, como a voltametria de pulso diferencial, além de também minimizar ao máximo os efeitos da corrente capacitiva. Além disto, observando-se o sinal de corrente obtidos nas varreduras diretas e inversas é possível conseguir as mesmas informações da voltametria cíclica e, assim, analisar o mecanismo de redução eletroquímica da ametrina pela variação dos parâmetros voltamétricos.

\section{Procedimento Experimental}

As análises voltamétricas foram realizadas em um potenciostato da Radiometer Analytical modelo PGZ 402, acoplado a um microcomputador AMD K6 dotado com o software Voltamaster, também da Radiometer Analytical.

Como eletrodo de trabalho foi utilizado um sistema de eletrodo de gota suspensa de mercúrio, modelo MDE 150 também da Radiometer Analytical, que utiliza como eletrodo de referência o eletrodo $\mathrm{Ag}$ / $\mathrm{AgCl} \mathrm{em} \mathrm{KCl} \mathrm{1,0} \mathrm{mol} \mathrm{L-1,} \mathrm{e} \mathrm{como} \mathrm{eletrodo} \mathrm{auxiliar}$ ou contra-eletrodo um fio de platina.

Todos os reagentes utilizados foram de grau analítico e as soluções foram preparadas em água Milli-Q. O pesticida utilizado foi gentilmente cedido pela Ciba GeigyÒ do Brasil.

\section{Resultados e discussão}

De acordo com dados da literatura [11], as s-triazinas hidrolisam em $\mathrm{pH}<2,0$. Assim, testes de eletroatividade em diferentes eletrólitos suporte foram realizados neste valor limite de $\mathrm{pH}$ visando selecionar aquele onde uma resposta ótima de corrente e de potencial de pico fosse obtida. Os eletrólitos utilizados foram: $\mathrm{Na}_{2} \mathrm{SO}_{4}, \mathrm{NaClO}_{4}, \mathrm{KCl}$ e tampão Britton Robinson todos na concentração de $0,1 \mathrm{~mol} \mathrm{~L}^{-1}$. Entretanto, em todos estes eletrólitos obteve-se uma resposta voltamétrica muito semelhante, o que indicou a pouca influência do eletrólito no processo eletroquímico. Assim, optouse por realizar todo o trabalho em meio de $\mathrm{Na}_{2} \mathrm{SO}_{4}$ $0,1 \mathrm{~mol} \mathrm{~L}^{-1}$.

\section{Variação do pH}

A redução voltamétrica da ametrina foi avaliada num intervalo de $\mathrm{pH}$ de 0,5 a 5,5 com os valores sendo ajustados com $\mathrm{H}_{2} \mathrm{SO}_{4} 0,1 \mathrm{~mol} \mathrm{~L} \mathrm{~L}^{-1}$, utilizando-se amostras preparadas a partir de uma solução estoque de ametrina dissolvida em uma mistura de água e etanol $20 \%$ v/v, para assegurar a solubilidade da ametrina.

Tanto na voltametria cíclica quanto na voltametria de onda quadrada, um único pico de redução foi observado, sendo este fortemente dependente do $\mathrm{pH}$ do meio.A Figura 2 apresenta os voltamogramas resultantes da variação do $\mathrm{pH}$ para uma solução de $1 \times 10^{-4} \mathrm{~mol} \mathrm{~L}^{-1}$ de ametrina em meio de $\mathrm{Na}_{2} \mathrm{SO}_{4} 0,1 \mathrm{~mol} \mathrm{~L}^{-1}$.

Avaliando-se o comportamento da corrente de pico em função do pH, observou-se

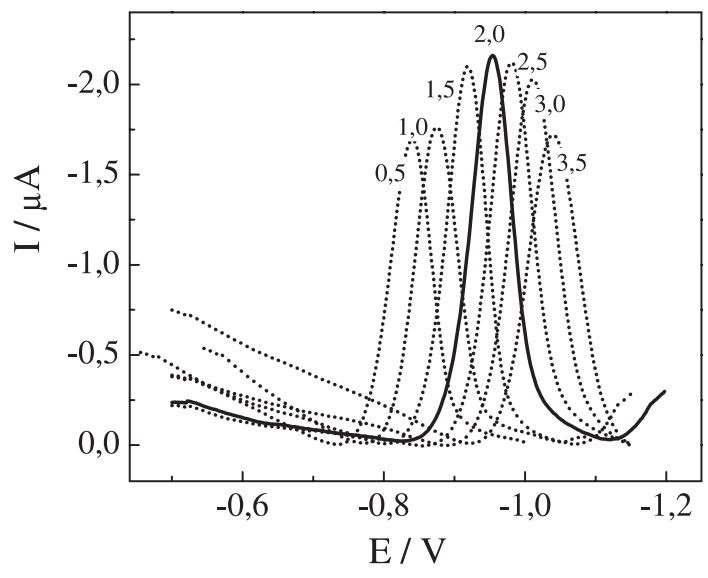

Figura 2. Voltamogramas de onda quadrada para a ametrina em meio de Na2SO4 0,1 mol L-1 com $a=50 \mathrm{mV}, \Delta E_{s}=2 \mathrm{mV}$, $f=100$ s-1 em função da variação do $\mathrm{pH}$ do meio. 
um máximo de corrente de pico em $\mathrm{pH}$ 2,0. Este máximo de corrente geralmente está associado à constante de ionização da espécie $\left(\mathrm{pK}_{\mathrm{a}}\right)$.

Em relação aos potenciais de pico, observou-se um deslocamento para valores mais negativos com o aumento do $\mathrm{pH}$ do meio, este comportamento é típico de processos onde ocorre prévia protonação da espécie. Este comportamento foi confirmado utilizando-se a inclinação da relação linear entre o potencial de pico e o pH, dada por:

$$
\mathrm{E}_{\mathrm{p}}=-0,811+67,3 \mathrm{pH}
$$

que, relacionando-se com a equação de Nernst (60 $\mathrm{mV}$ de inclinação para a transferência de $\mathrm{n}$ prótons e $n$ elétrons, com $n=1,2,3$. .) [12], mostra a possibilidade de envolvimento do mesmo número de prótons e elétrons na redução eletroquímica. A Figura 3 apresenta as relações entre a corrente e potencial de pico com a variação do $\mathrm{pH}$ do eletrólito suporte.

Assim o pH definido como aquele que apresenta uma melhor resposta para estudo

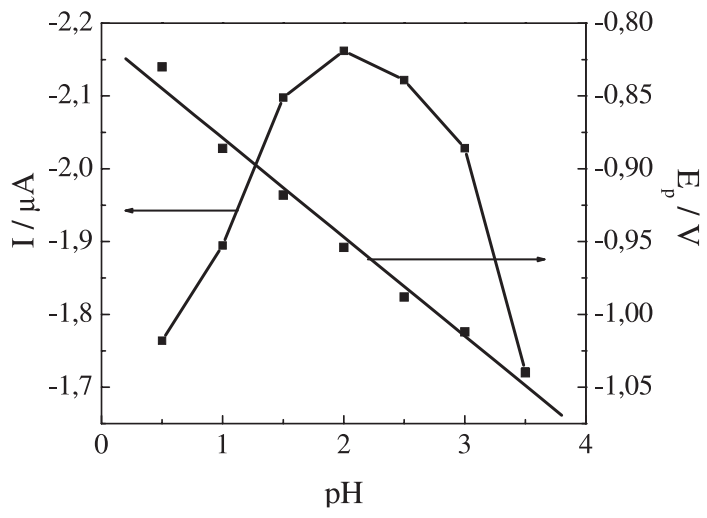

Figura 3. Relação da corrente e potencial de pico em função da variação do $\mathrm{pH}$ do meio, com os mesmos parâmetros da Figura 2.

eletroquímico da ametrina foi 2,0 valor no qual, o potencial de pico é $-0,93 \mathrm{~V}$ vs. $\mathrm{Ag} / \mathrm{AgCl}$.

Componentes de corrente

Os parâmetros da SWV também foram avaliados a fim de se obter os valores otimizados para o estudo eletroquímico da ametrina. A separação das componentes de corrente direta, reversa e resultante mostrou que a corrente direta é praticamente coincidente com a corrente resultante, comportamento típico de processos totalmente irreversíveis. A Figura 4 apresenta a separação das componentes de corrente para a ametrina.

\section{Variação da freqüência de aplicação dos pulsos}

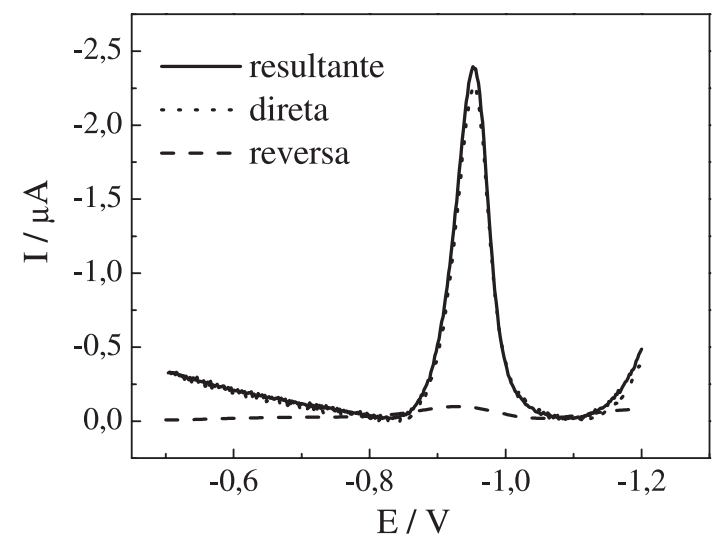

Figura 4. Voltamogramas de onda quadrada para a ametrina em meio de Na2SO4 0,1 mol L-1 com $a=50 \mathrm{mV}, \Delta E_{s}=2 \mathrm{mV}$, $f=100 \mathrm{~s}-1$ e pH 2,0 com a separação das componentes de corrente direta, reversa e resultante.

\section{de potencial ( $f$ )}

A freqüência de aplicação de pulsos de potencial $(f)$ é um dos mais importantes parâmetros voltamétricos envolvidos na SWV, pois além de melhorar a sensibilidade da análise ainda possibilita uma observação do tipo de processo e o número de elétrons envolvidos na transferência eletrônica. A Figura 5 apresenta os voltamogramas obtidos em diferentes valores de frequiência de aplicação dos pulsos de potenciais para a ametrina, até valores de $100 \mathrm{~s}^{-1}$, acima deste valor o aumento da corrente em função da freqüência não promove melhora significativa nas respostas voltamétricas.

Para a ametrina o aumento da frequiência de aplicação dos pulsos de potencial promoveu um 


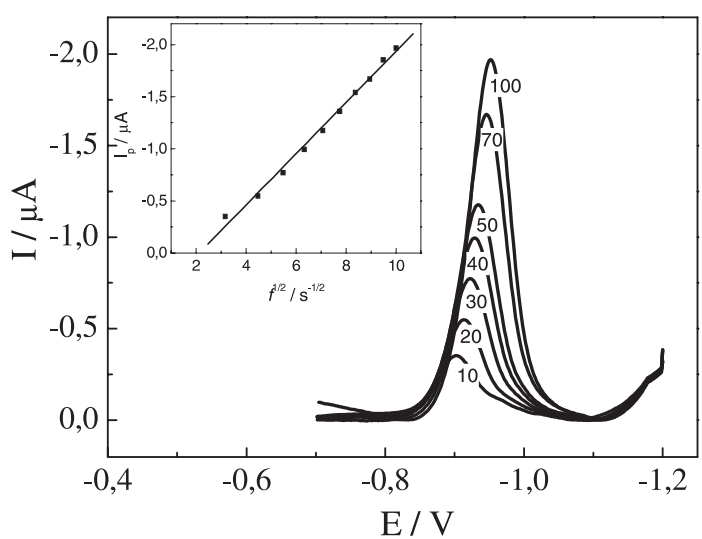

Figura 5. Voltamogramas de onda quadrada para a ametrina em Na2SO4 0,1 mol L-1 com $a=50 \mathrm{mV}, \Delta E_{\mathrm{s}}=2 \mathrm{mV}, 1 \mathrm{e} \mathrm{pH}$ 2,0 em função da variação de freqüência de aplicação dos pulsos de potencial, com a inserção da relação da corrente de pico com a raiz quadrada da freqüência.

aumento linear da corrente de pico em função da raiz quadrada da freqüência. De acordo com os critérios de diagnóstico da SWV [13, 14], este comportamento é típico de processos controlados pela difusão do reagente. A inserção na Figura 5 apresenta esta relação. Este comportamento foi confirmado com dados de voltametria cíclica, onde a relação linear do logaritmo da corrente de pico em função do logaritmo da velocidade de varredura apresenta uma inclinação de 0,6 valor este característico de processos difusionais.

De acordo com os critérios da SWV, neste tipo de sistema os potenciais de pico variam linearmente com o logaritmo da frequiência, com uma inclinação dada pela Equação de Lovric [13]:

$$
\frac{E_{p}}{\Delta \log f}=-\frac{2,3 R T}{\alpha n F}
$$

onde R é a constante dos gases, $\mathrm{T}$ é a temperatura, a é o coeficiente de transferência eletrônica, $n$ o número de elétrons envolvidos na transferência eletrônica e F a constante de Faraday. Assim, para a ametrina a inclinação obtida foi $58 \mathrm{mV}$ que, aplicando na Equação 2, fornece um valor de an igual a 1 . Se considerarmos que o valor de $a$ é 0,5 , valor comumente utilizado, o número de elétrons envolvidos na transferência eletrônica é 2, o que pode estar relacionado à redução do grupo tiometila $\left(-\mathrm{SCH}_{3}\right)$ após a monoprotonação de cada um dos grupos amina da molécula. A Figura 6 apresenta um possível mecanismo para a redução da ametrina. Variação da amplitude dos pulsos (a)

Para reações redox totalmente irreversíveis a sensibilidade analítica na SWV sofre uma grande

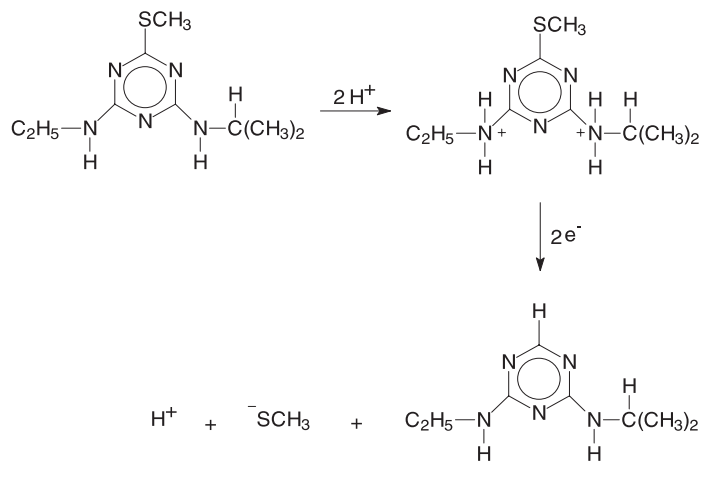

Figura 6. Proposta de mecanismo de redução da ametrina.

influência do aumento da amplitude da onda, isto porque a largura de meia-onda se mantém constante para amplitudes maiores que $20 \mathrm{mV}^{4}$. Com isso, a variação na amplitude de pulso é um dos parâmetros a ser otimizado na análise por SWV.

Para a ametrina, a variação na amplitude dos pulsos de potencial provocou um aumento na intensidade das correntes de pico e um deslocamento dos potenciais para valores mais positivos. A Figura 7 apresenta os voltamogramas resultantes da variação de amplitude de pulsos de potencial para a ametrina.

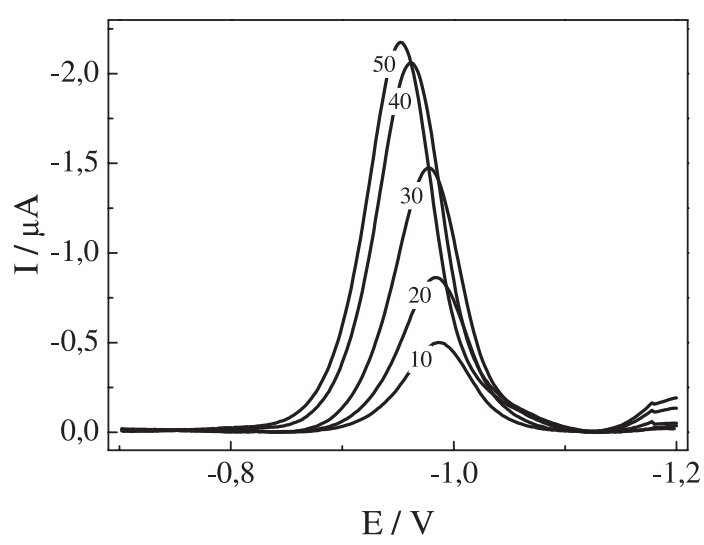

Figura 7. Voltamogramas de onda quadrada para a ametrina em $\mathrm{Na} 2 \mathrm{SO} 40,1 \mathrm{~mol} \mathrm{~L}-1$ com $a=50 \mathrm{mV}, \Delta E_{s}=2 \mathrm{mV}, 1 \mathrm{e} \mathrm{pH} \mathrm{2,0} \mathrm{em}$ função da variação da amplitude dos pulsos de potencial. 
Uma análise do comportamento da corrente de pico em função das mudanças na amplitude dos pulsos de potenciais mostrou que um aumento linear até amplitude de $40 \mathrm{mV}$, após este valor o aumento lão é mais linear, tendendo a se tornar constante com o aumento da amplitude, evidenciando um patamar. Este comportamento está de acordo com a teoria da SWV, desenvolvida para sistemas totalmente irreversíveis com a espécie adsorvida na superfície do eletrodo, que afirma que para amplitudes acima de $50 \mathrm{mV}$ a corrente de pico é constante [3].

\section{Variação do incremento de potencial $\left(\mathrm{DE}_{\mathrm{s}}\right)$}

O incremento de potencial é um dos responsáveis pela definição da velocidade de varredura de potenciais, já que esta é dada pelo produto do incremento de potencial pela freqüência de aplicação dos pulsos de potencial. Assim, um aumento do incremento de potencial deveria significar uma melhora na sensibilidade das análises, contudo, a variação do incremento de varredura não forneceu melhora significativa nas respostas voltamétricas, isto pode estar relacionado a alguma limitação do equipamento utilizado. Assim, o valor de $D E_{s}$ utilizado foi de $2,0 \mathrm{mV}$.

Curvas analíticas para análise da ametrina

Após a otimização das condições experimentais e voltamétricas, voltamogramas de onda quadrada foram obtidos para a redução da ametrina em diferentes concentrações em meio de $\mathrm{Na}_{2} \mathrm{SO}_{4} 0,1 \mathrm{~mol} \mathrm{~L}^{-1} \mathrm{pH} 2,0$. Estes voltamogramas foram obtidos utilizando-se o método de adição padrão em soluções preparadas em água pura de laboratório.

Nestas condições, observou-se um aumento linear da corrente de pico com a adição de ametrina. A Figura 8 apresenta os voltamogramas obtidos com as adições de ametrina no intervalo de concentração de: $1,0 \times 10^{-7}$ a $1,2 \times 10^{-}$ ${ }^{5} \mathrm{~mol} \mathrm{~L}^{-1}$, e a Figura 9 apresenta a relação entre as correntes de pico e a concentração de ametrina.

Para a determinação do limite de detecção utilizou-se o desvio padrão da média aritmética das correntes obtidas de seis voltamogramas de brancos, no mesmo potencial do pico voltamétrico de redução da ametrina, e a relação [15]:
$L D=\frac{3 S_{B}}{b}$

onde $\mathrm{S}_{\mathrm{B}}$ é o desvio padrão da média aritmética das correntes obtidas dos brancos e b o valor do coeficiente angular da curva analítica. $\mathrm{O}$ valor calculado para a ametrina foi $4,39 \times 10^{-10} \mathrm{~mol} \mathrm{~L}^{-1}$ que corresponde à $0,1 \mathrm{mg} \mathrm{L}^{-1}(0,1 \mathrm{ppb})$, considerando-se dados da Figura 9.

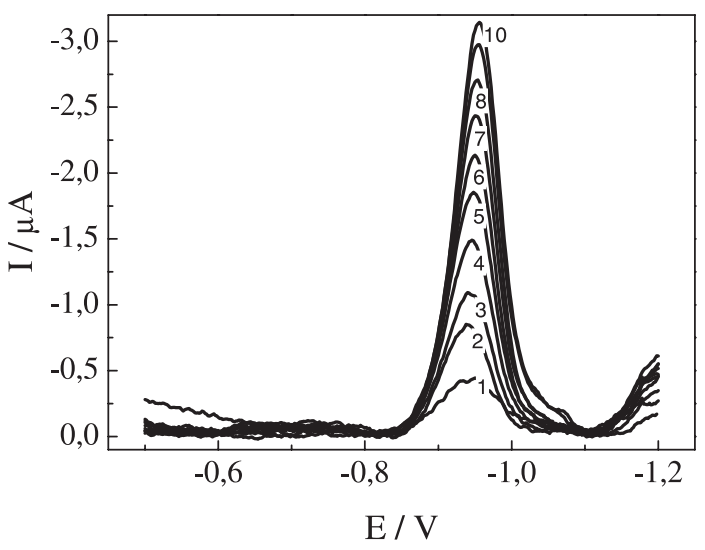

Figura 8. Voltamogramas de onda quadrada para a ametrina em meio de Na2SO4 0,1 mol L-1 com $a=50 \mathrm{mV}, \Delta E_{s}=2 \mathrm{mV}, 1 \mathrm{e}$ $\mathrm{pH} 2,0$, onde: (1) $9,71 \times 10^{-7}$; (2) $1,94 \times 10^{-6}$; (3) $2,86 \times 10^{-6}$; (4) $3,87 \times 10^{-6}$; (5) $4,84 \times 10^{-6}$; (6) $5,81 \times 10^{-6}$; (7) $6,77 \times 10^{-6}$; (8) $7,58 \times 10^{-6}$; (9) $8,55 \times 10^{-6}$; (10) $8,55 \times 10^{-6} \mathrm{~mol} \mathrm{~L}^{-1}$ de ametrina.

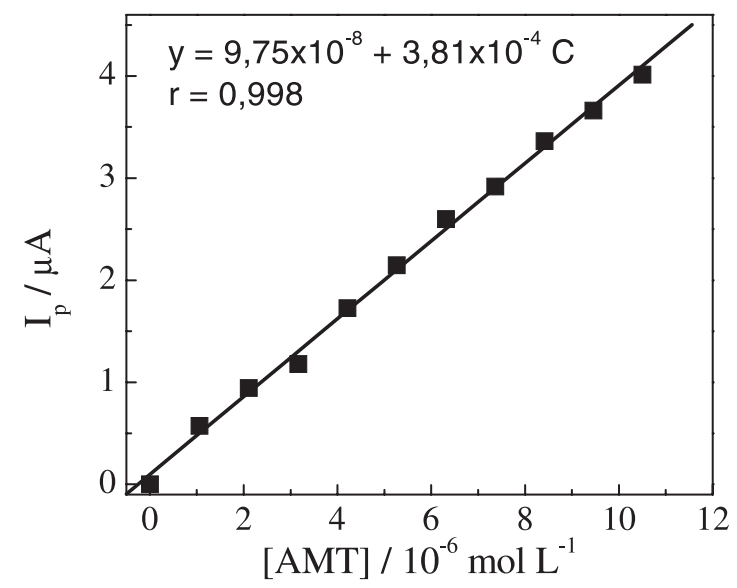

Figura 9. Relação da corrente de pico com a concentração da ametrina, com os dados da Figura 8. 


\section{Conclusões}

A utilização da SWV para o estudo do comportamento eletroquímico da ametrina sobre HMDE possibilitou a constatação de que este processo envolve dois elétrons numa redução totalmente irreversível, onde ocorre protonação prévia da espécie. Uma proposta preliminar de mecanismo de redução considera a redução do grupo tiometila do anel aromático, após a protonação dos grupos amina, que serve para desestabilizar a molécula do reagente.

A dependência do sinal analítico com a concentração do pesticida em solução permitiu a determinação de um limite de deteç̧ão ótimo para análises de traços, com um valor de $0,1 \mathrm{mg} \mathrm{L}^{-1}(0,1 \mathrm{ppb})$.
Posterior desenvolvimento para esta metodologia deve envolver a utilização de técnicas de redissolução e aplicação para amostras naturais de água, solos ou alimentos, assim como a utilização de eletrólises a potenciais controlados para a determinação dos produtos da reação, visando validar (ou modificar) o mecanismo sugerido.

\section{Agradecimentos}

Os autores agradecem ao CNPq (Proc. 478263/01-5) e FAPESP (Proc. 99/07891-9) pelos auxílios financeiros e bolsas concedidas durante a realização deste trabalho.

M. F. Cabral, D. de Souza, C. R. Alves, S. A. S. Machado. Square wave voltammetric study of the electrochemical behavior of the herbicide ametryne.

Abstract: The determination of the herbicide ametryne in acid solution was performed using square
wave voltammetry and a static drop mercury electrode. The electroredution reaction proceeds through a
completely irreversible mechanism with the diffusion of the electroactive specie being the rate determining
step. It was observed a previous protonation of the reagent molecule followed by the transference of two
electrons. The linearity of peak current with concentration allows to calculate a detection limit of $0,1 \mathrm{mg}$
$\mathrm{L}^{-1}(0,1 \mathrm{ppb})$. This value is adequate for the analysis of herbicide contamination (e.g., in drinking water).

Keywords: ametryne; square wave voltammetry; mercury electrode.

\section{Referências bibliográficas}

[1] V. Pacáková, K. Stulik, J. Jiskra. .J. Chromatogr., A, 754 (1996) 17.

[2] L. M. Ignjatovic, D. S. Veselinovic, D. A. Markovic, N. S. Vukelic. J. Serb. Chem. Soc. 63 (1998) 75.

[3] J. Osteryoung, R. Osteryoung. Anal. Chem. 57 (1985) 101'.

[4] D. Souza, S. A. S. Machado, L. A. Avaca. Quím. Nova 26 (1) (2003) 81.

[5] L. Codognoto, S. A. S. Machado, L. A. Avaca. Diamond Relat. Mater. 11 (2002) 1670.

[6] D. Souza, S. A. S. Machado. Quím. Nova, no prelo.

[7] J. M. Zen, S. H. Jeng, H. J. Chen. Anal. Chem. 68 (1996) 498.

[8] A. Walcarius, L. Lamberts. J. Electroanal. Chem. 406
(1996) 59.

[9] R. J. Hance. Pestic. Sci.. 1 (1979) 112.

[10] H. Benadikova, R. Kalvoda. Anal. Lett. 17 (A13)(1984) 1519 .

[11] L. M. Ignjatovic, D. A. Markovic, D. S. Veselinovic. Electroanalysis 5 (1993) 529.

[12] C. M. P. Vaz, S. Crestana, S. A. S. Machado, L. H. Mazo, L. A. Avaca. Int. J. Environ. Anal. Chem..62 (1996) 65.

[13] M. Lovriæ, S. Komorsky-Lovric, R. W. Murray. Electrochimic. Acta 33 (1988) 739.

[14] M. Lovriæ, S. Komorsky-Lovric. J. Electroanal. Chem. 248 (1988) 239.

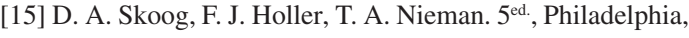
Saunders Golden Sunburst Series, 1998. 